\title{
Validity of endocan as a biomarker in systemic sclerosis: relation to pathogenesis and disease activity
}

\author{
Marwa Yahia Mahgoub ${ }^{1 *}$ (D) Ali Ibrahim Fouda', Ahmed Yousef Elshambaky ${ }^{1}$, Walaa Bayoumie Elgazzar ${ }^{2}$ and \\ Sara Abdellatif Shalaby ${ }^{3}$
}

\begin{abstract}
Background: Systemic sclerosis (SSc), an autoimmune disease, has endothelial dysfunction and tissue fibrosis. The lack of its specific activity markers urges the research. Endocan is an immunomodulatory protein associated with endothelial dysfunction. We aimed to measure of the endocan level in systemic sclerosis (SSc) patients' sera to test its relation to disease activity and treatment responses. Also, to evaluate its specificity by comparing these data with Behçet's disease (BD) and rheumatoid arthritis (RA) patients' data.

Results: SSC and RA patients were in activity but in the BD group; eight were active and seven were inactive. The endocan level was higher in the three groups in comparison to the controls without statistically significant differences ( $p$ value RA 0.697, SSC 0.063, and BD 0.196). A statistically significant difference in endocan levels between SSc patients with and without vascular manifestations (pulmonary hypertension and finger pitting ulcers, $p<0.0001, p=0.0097$, respectively). There were no significant correlations between endocan level and the erythrocyte sedimentation rate and $\mathrm{C}$-reactive protein (positive rheumatoid factor for RA) in the three groups $(p>0.05)$. There were no significant correlations between endocan level and activity scores in the three groups $(p>0.05)$. The cut-off value of endocan was $1.3 \mathrm{ng} / \mathrm{ml}$.
\end{abstract}

Conclusion: Endocan is a non-specific marker for the vascular pathogenesis in systemic sclerosis but not a good predictor for its activity.

Keywords: Endocan, Systemic sclerosis, Rheumatoid arthritis, Behcet's disease

\section{Background}

The functions of endothelial cells (ECs) in healthy microenvironment include separation of blood contents from the interstitial space, prevention of platelet aggregation, inhibition of coagulation cascade, and modulation of the state of smooth muscle contraction (vascular tone). ECs synthesize and secrete various substances to mediate these multiple functions [1].

Endocan, known as endothelial cell-specific molecule1 (ECSM1), is a proteoglycan expressed by ECs. It is a

\footnotetext{
* Correspondence: marwayahiamlm@gmail.com

${ }^{1}$ Rheumatology, Rehabilitation and Physical Medicine Department, Benha University, Benha 13115, Egypt

Full list of author information is available at the end of the article
}

common molecular property in vascular tissues, like capillaries, arterioles, and venules. The main function of the endocan is inhibiting the interaction between intercellular adhesion molecule- 1 and the integrin on lymphocytes and monocytes. Therefore, it affects integrinmediated leukocyte functions, as the firm adhesion of leukocytes to the endothelium and the leukocyte migration itself into tissues. Numerous inflammatory diseases were studied for the endocan level and some studies showed its relation to their pathogenesis [2].

Systemic sclerosis (SSc) is an autoimmune disease with complex pathogenesis. The cornerstones of that pathogenesis are endothelial injury and autoimmunity, which are associated with interstitial and vascular fibrosis [3]. 
These intricated pathogenesis and disease course increase the need for validated biomarkers for diagnosis, disease classification, and evaluation of disease activity [4]. High serum vascular endothelial growth factor levels were detected in patients with SSc, which were demonstrated to upregulate endocan and its transcription gene [5]. This research aimed to analyze the real relationship between endocan with SSc and its activity.

\section{Methods}

This case-control study was conducted at our university, Faculty of Medicine, Egypt, as a collaborative intradepartmental project between the Departments of Rheumatology, Rehabilitation and Physical Medicine and Medical Biochemistry, and Molecular Biology.

Group of SSc included 15 patients who were diagnosed according to 2013 ACR/EULAR criteria [6] .They were women and their age ranged between 25 and 55 years. Group of Behçet's disease (BD) were 15 patients diagnosed according to the International Criteria for Behçet's Disease 2006 [7]; all of them were men and their age ranged from 25 to 50 years. Group of Rheumatoid Arthritis (RA) involved 30 patients diagnosed according to the 2010 ACR/EULAR criteria [8]. They were 20 women and 10 men, and their age ranged from 23 to 75 years. Thirty healthy individuals were involved as controls. Eleven men participated as a separate control group to BD group. Fifteen females of the control were a separate control group to SSc group. All the 30 controls were a control group of RA group. Their range of age was between 20 and 60 years.

All patients were recruited from the inpatients' and the outpatients' clinic of Rheumatology, Rehabilitation, and Physical Medicine Department, in our University Hospitals. All the patients in the study signed a detailed written consent form in Arabic. Full history-taking, and general and systemic examinations were considered for all groups.

\section{Assessment of disease activity}

The European Scleroderma Study Group/EUSTAR activity score [9] was followed to define disease activity criteria for SSc. Its components are modified Rodnan score, deterioration of skin in previous month, deterioration of vascular (Raynauds)/GIT in previous month, digital necrosis, arthritis, DLCO $<80 \%$, deterioration in heart-lung in previous month, ESR > $30 \mathrm{~mm}$ in first hour, and hypocomplementemia. The disease is considered active if the sum of the scores of the detected items is more than or equal to 3. All patients of SSc were in active disease.

Behçet's Disease Current Activity Form 2006 [10] was used to evaluate BD patients. The score is out of 12 which represents the Disease Activity Index. Of the BD patients, eight were active and seven were of inactive disease.

RA disease activity was assessed using the modified disease activity score (DAS28) [11]. All patients of RA were in active disease.

\section{Sampling and biochemical analysis}

About $10 \mathrm{ml}$ of venous blood was withdrawn from each participant under complete aseptic conditions. One part about $3 \mathrm{ml}$ was taken into the vacutainer EDTA tube. The resting part $(7 \mathrm{ml})$ was left to be clotted at room temperature for $15-30 \mathrm{~min}$ and centrifuged at $3000 \times g$, for $10 \mathrm{~min}$ to separate the serum. Separated serum samples were collected in Eppendorf tubes and stored at $80{ }^{\circ} \mathrm{C}$ until used for spectrophotometric measurements and endocan level determination by the enzyme-linked immunosorbent assay (ELISA).

The following hematological and biochemical investigations were done: complete blood count via a Sysmex analyzer (XN-100), rheumatoid factor using the rheumatoid factor latex kit (Diagnostic Automation, the Netherlands), C-reactive protein (CRP) examined via rapid CRP kit (OXOID, Ontario, Canada), and erythrocyte sedimentation rate (ESR) according to Westergren method and the values were expressed $(\mathrm{mm} / \mathrm{h})$ : less than $15 \mathrm{~mm} / \mathrm{h}$ was considered to be normal, anti-scleroderma 70 using Scl-70 ELISA kit (Diagnostic Automation). Other routine biochemical tests including serum creatinine, blood urea nitrogen, serum aspartate aminotransferase, and serum alanine aminotransferase were analyzed using kits obtained from Biosystems (Spain). They were measured by spectrophotometer UVD3200 (Labomed Inc., USA).

\section{Serum endocan measurement}

The serum endocan level was determined by the ELISA according to the manufacturer's protocol (Human ECSM1/ENDOCAN ELISA Kit, Catalog No.: 201-121978; Sunred Biological Technologies, China). A measure of $40 \mu \mathrm{l}$ of each serum sample, having the ECSM1/ endocan, was added to the monoclonal antibody enzyme well which is precoated with human ECSM1/endocan monoclonal antibody and then $10 \mu \mathrm{l}$ of ECSM1/endocan antibodies labeled with biotin, and $50 \mu \mathrm{l}$ of streptavidinHRP was added to form an immune complex and incubated for $60 \mathrm{~min}$ at $37^{\circ} \mathrm{C}$ followed by washing to remove the uncombined enzyme. A measure of $50 \mu$ l chromogen solution A and then $50 \mu \mathrm{l}$ chromogen solution B were added to each well, gently mixed, and incubated for 10 min at $37{ }^{\circ} \mathrm{C}$ away from light. Finally, $50 \mu \mathrm{l}$ of the stop solution was added into each well to stop the reaction (the blue color formed after the addition of the chromogen solutions changed into yellow immediately). Optical density was measured under $450 \mathrm{~nm}$ wavelength within 
15 min after adding the stop solution using the ELISA reader (Infinite F50; TECAN, GmbH).

\section{Statistical analysis}

SPSS statistical software was used for all statistical analyses (IBM SPSS statistics, version 21.0; SPSS Inc.). The data were normally distributed. Numerical data were expressed as mean value \pm SD while categorical data were presented as numbers and percentages. Variations among groups in clinical and serological data were linked by the $t$ test for normally distributed values. $p$ value $\leq 0.05$ was considered statistically significant.

\section{Results}

Our patients were grouped into SSc group which included 15 female patients of SSc (mean age, $38.60 \pm$ 0.18 ) with mean disease duration 10 months .The BD group included 15 male patients of BD (mean age, 37.87 \pm 9.36) with mean disease duration 9 months, and the RA group included 30 (20 female and 10 male) patients of RA (mean age, $43.97 \pm 13.83$ ) with mean disease duration 7.9 months. Control groups included 30 healthy individuals (mean age, $39.83 \pm 11.12$ ) with no statistically significant difference between the three groups and control groups. Clinical manifestations of SSc patients are shown in Table 1.

\section{Endocan level results}

There were no statistically significant differences between any disease group and its control group $(p<0.05)$ as in Table 2.There were no significant differences between endocan levels when were compared between SSc patients and neither RA nor BD patient's sera levels ( $p=$ 0.0845 and $p=0.0253$, respectively).

Although there was a non-significant increase in endocan level in the SSc group than its control group, SSc patients with and without vascular manifestations showed a statistically significant difference in endocan levels (pulmonary hypertension $p<0.0001$ and finger

Table 1 Systemic sclerosis patients' clinical data

\begin{tabular}{ll}
\hline Clinical findings & $n(\%)$ \\
\hline Diffuse skin tightness & $11(73.3)$ \\
Limited skin tightness & $4(26.6)$ \\
Raynaud's disease & $15(100)$ \\
GIT involvement & $10(66.6)$ \\
Pulmonary hypertension & $4(26.6)$ \\
Calcinosis & $2(13.3)$ \\
Fingertip pitting ulcers & $8(53.3)$ \\
Interstitial lung disease & $5(33.3)$ \\
Arthritis & $7(46.6)$ \\
\hline
\end{tabular}

GIT gastrointestinal tract
Table 2 Serum endocan level in RA, SSC, BD, and control groups

\begin{tabular}{lllll}
\hline Serum endocan $(\mathrm{ng} / \mathrm{ml})$ & Range & Mean $\pm \mathrm{SD}$ & $t$ test & $p$ value \\
\hline RA & $1.01-3.07$ & $1.35 \pm 0.16$ & 0.391 & 0.6972 \\
Control & $1.02-2.26$ & $1.37 \pm 0.23$ & & \\
SSC & $1.03-3.05$ & $1.43 \pm 0.10$ & -1.934 & 0.0633 \\
Control & $1.02-2.06$ & $1.34 \pm 0.15$ & & \\
BD & $1.02-2.04$ & $1.32 \pm 0.15$ & -1.322 & 0.1967 \\
Control & $1.04-2.26$ & $1.40 \pm 0.18$ & &
\end{tabular}

$B D$ Behçet's disease, RA rheumatoid arthritis, SSc systemic sclerosis, $t$ test, Student's test

$p \leq 0.05$ is significant, $p>0.05$ is insignificant, $p$ value equal to $0.001=$ highly significant

pitting ulcers $p=0.0097$, respectively) (Table 3, Figs. 1 and 2).

On the one hand, there was no significant difference demonstrated between the endocan levels in patients with and without vascular manifestation neither in BD group nor in RA group (Tables 4 and 5).

\section{Endocan level and diseases activity}

For SSc (European Scleroderma Study Group/EUSTAR score), all patients were in activity (100\%) (mean $6.26 \pm$ 1.9), and there was no significant correlation between serum endocan level and the activity score $(r=0.071, p$ $>0.05)$. Regarding the DAS28 of RA, all patients were active (mean $5.35 \pm 1.3$ ), and there was no significant correlation between serum endocan level and the activity score $(r=0.034, p>0.05)$. Regarding score activity of BD disease (mean $11.38 \pm 5$ ), $53.3 \%$ of patients were active and $46.7 \%$ were inactive, and serum endocan level showed no statistically significant difference between patients in activity $(1.26 \pm 0.32 ; 1.02-2.04 \mathrm{ng} / \mathrm{ml})$ and those showed no activity $(1.41 \pm 0.39 ; 1.09-2.04 \mathrm{ng} / \mathrm{ml})$ $(p>0.05)$.

The acute-phase reactants (ESR, CRP) had a statistically highly significant difference between each group and the control group $(p=0.001)$, but there was no significant correlation between serum endocan levels and acute-phase reactant parameters in the three groups (RA, SSc, BD) $(p>0.05)$.

Table 3 Endocan levels in patients regarding vascular manifestations of SSc patients

\begin{tabular}{lllll}
\hline Vascular manifestations & & Mean \pm S. D & t. test & $p$ value \\
\hline Pulmonary hypertension & +ve & $2.85 \pm 0.22$ & 1.864 & $p<0.0001$ \\
& $-v e$ & $0.39 \pm 0.2$ & & \\
Finger pitting ulcers & + ve & $2.35 \pm 0.88$ & 1.064 & $p=0.0097$ \\
& -ve & $0.92 \pm 0.32$ & & \\
\hline
\end{tabular}

SSc systemic sclerosis, $t$ test Student's test, $p$ value probability value, SD standard deviation, $p \leq 0.05$ is significant, $p>0.05$ is insignificant, $p=0.001$ highly significant 


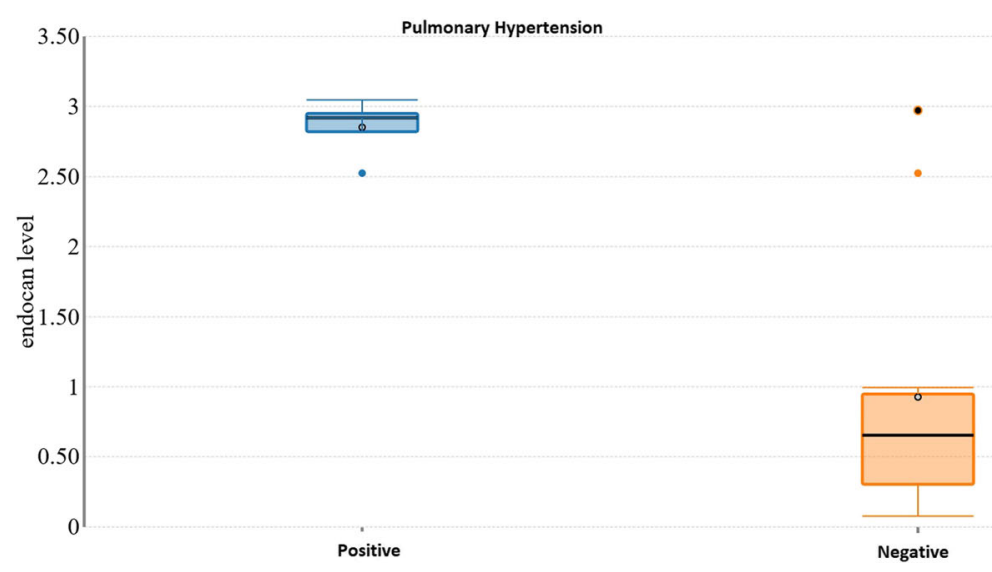

Fig. 1 Box plot of endocan level in patients with and without pulmonary hypertension

\section{Endocan level and treatment lines}

There were $60 \%$ of RA patients on methotrexate (MTX) with steroid and $40 \%$ were on MTX and hydroxychloroquine. Eighty percent of the SSc patients were on symptomatic treatment and DMARD and 20\% were on symptomatic treatment and cyclophosphamide. About $53.3 \%$ of BD patients were on biological therapy and $46.7 \%$ were on immunosuppressive therapy. There was no statistically significant difference between any line of treatment and serum endocan level in the three groups $(p>0.05)$.

\section{Discussion}

Many cytokines were proved to have influences on the clinical presentation of systemic sclerosis patients as serum IL-27 that is associated with significant cutaneous and pulmonary involvement [12]. ECSM1 (endocan) is an important immunomodulatory protein secreted by ECs. The last years of research accentuated its role in the molecular mechanisms underlying the pathogenesis of several diseases with endothelial dysfunction [13-15].

This study aimed to evaluate endocan sensitivity in SSc pathogenesis and disease activity. RA and BD patients were involved in the study as autoimmune diseases are known to have endothelial dysfunction in their pathogenesis $[16,17]$ for comparison and specificity assessment.

In SSc patients, the level of endocan was slightly higher than the control group which matches the results of Bălănescu et al. [18] but still with a statistically insignificant difference. This may be clarified by several explanations. The first is that at the time of sampling, most of SSc patients suffer from Raynaud's disease and/ or pulmonary hypertension and were treated by antihypertensive drugs. Many researchers approved that serum endocan level was downregulated by antihypertensive medications [19]. The second explanation that some of the SSc patients had more predominant fibrotic pathogenesis (skin and gastrointestinal involvement) than

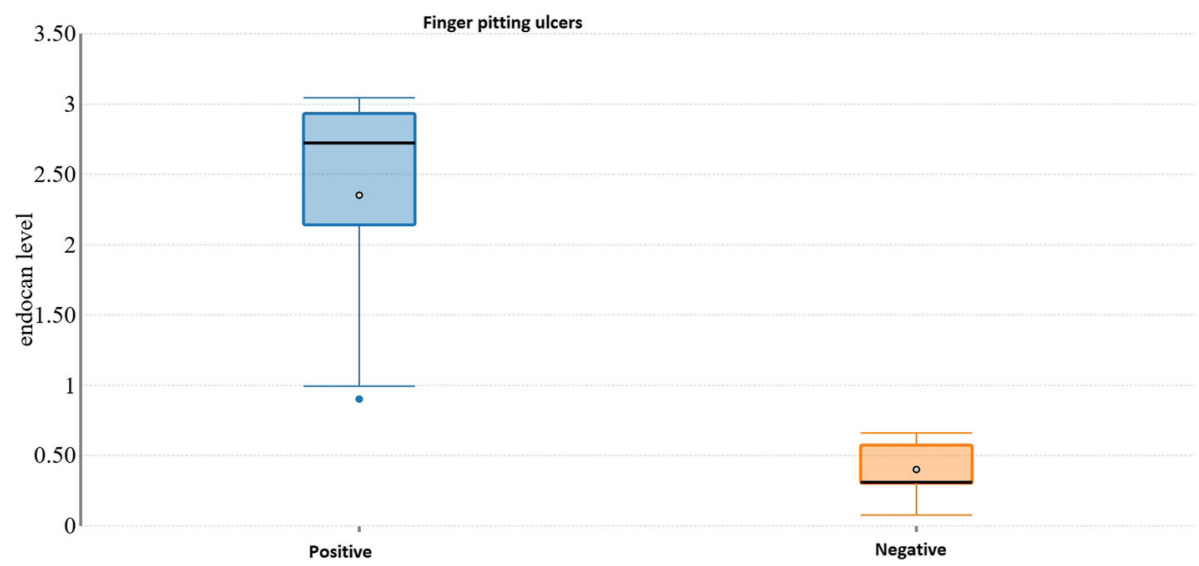

Fig. 2 Box plot of endocan level in patients with and without finger pitting ulcers 
Table 4 Endocan levels in patients regarding vascular manifestations of $\mathrm{BD}$ patients

\begin{tabular}{lllll}
\hline Vascular manifestations & & Mean \pm SD & $t$ test & $p$ value \\
\hline Genital ulcers & +ve & $1.36 \pm 0.37$ & 0.864 & 0.370 \\
& -ve & $1.12 \pm 0.04$ & & \\
Erythema nodosum & + ve & $1.35 \pm 0.38$ & 0.064 & 0.804 \\
& -ve & $1.30 \pm 0.32$ & & \\
Papulopustular lesions & +ve & $1.40 \pm 0.39$ & 1.801 & 0.203 \\
& -ve & $1.13 \pm 0.03$ & & \\
Uveitis & + ve & $1.36 \pm 0.37$ & 0.864 & 0.370 \\
& -ve & $1.12 \pm 0.04$ & & \\
\hline
\end{tabular}

$B D$ Behçet's disease. $t$ test Student's test, $p$ value probability value, $S D$ standard deviation, $p \leq 0.05$ is significant, $p>0.05$ is insignificant, $p=0.001$ highly significant

vascular dysfunction; with a limited role of endocan in fibrosis [20]. The third explanation is the small number of patients that reflects the uncommonness of that disease especially that the patients were recruited from one health opportunity that may suppose our results to type two statistical error. The insignificant difference of endocan levels between SSc, RA, and BD patients designates the specificity of endocan to SSc disease and increases the suggestion of involvement of endocan in different diseases with endothelial dysfunction pathogenesis.

However, there was a clearly significant difference in endocan levels being higher in SSc patients with pulmonary hypertension, and those had finger pitting ulcers than SSc patients who had any of these vascular signs. The elevation of serum endocan levels with these manifestations support the suggestion of the role of endocan in the underline vascular pathogenesis of SSc disease. Many studies reported the relation between endocan and the vascular dysfunction in variant diseases $[18,21$, 22].

Although many studies correlated increased serum endocan with inflammation, we noticed that there was no statistically significant difference between EUSTAR score activity nor acute-phase reactants (ESR and CRP) and endocan level in SSc patients. On the other hand, Tok et al. [20] concluded a negative correlation between endocan level and inflammation stage of chronic

Table 5 Endocan levels in patients regarding vascular manifestations of RA patients

\begin{tabular}{lllll}
\hline Vascular manifestations & & Mean \pm SD & $t$ test & $p$ value \\
\hline Vasculitis & +ve & $1.2 \pm 0.23$ & 0.765 & 0.245 \\
& -ve & $1.1 \pm 0.05$ & & \\
Raynaud's phenomenon & +ve & $1.27 \pm 0.24$ & 0.075 & 0.740 \\
& -ve & $1.30 \pm 0.31$ & & \\
\hline
\end{tabular}

$R A$ rheumatoid arthritis, $t$ test Student's test, $p$ value probability value, $S D$ standard deviation, $p \leq 0.05$ is significant, $p>0.05$ is insignificant, $p=0.001$ highly significant hepatitis which may support our remarks. Also, we revealed no statistically significant correlations between endocan level and the different lines of treatment in SSc patients which matched the findings of Bălănescu et al. [17].

In comparison with the RA group and BA group, we found no statistically significant difference between them and SSc group regarding the serum endocan level. It is worth to note that there was no correlation between the activity of both diseases and the endocan level.

Our study's limitations were the small sample number of patients as they were recruited from one hospital. The vacancy of SSc group and RA group from inactive cases that made us unable to obtain the statistical relation between them and the serum endocan level.

\section{Conclusion}

We concluded that serum endocan is a potential marker for the vascular pathogenesis incidence of systemic sclerosis but not a good predictor for its activity.

\section{Abbreviations}

SSc: Systemic sclerosis; BD: Behçet's disease; RA: Rheumatoid arthritis; ECs: Endothelial cells; ECSM1: Endothelial cell-specific molecule-1;

DLCO: Diffusing capacity of lung for carbon monoxide; MTX: Methotrexate

\section{Acknowledgements}

We acknowledge all the participants and medical staff who helped us in this study.

\section{Authors' contributions}

M.M: conception, design of the work, interpretation of data, and corresponding author. A.F: participation in analysis, interpretation of data, and revision. A.A: participation in analysis, interpretation of data, and revision. W.A: the laboratory work and writing of the laboratory practical part. S.S: collecting clinical data and statistical revision. All authors read and approved the submitted version (and any substantially modified version that involves the author's contribution to the study).

\section{Funding}

No funding opportunity.

\section{Availability of data and materials}

Data and materials are available with the corresponding author upon reasonable demand.

\section{Ethics approval and consent to participate}

This study was approved by the ethics committee of Benha University with approval number [16-12-20]. The written consent was obtained from study participants.

\section{Consent for publication}

Not applicable

\section{Competing interests}

The authors declare that they have no competing interests.

\section{Author details}

${ }^{1}$ Rheumatology, Rehabilitation and Physical Medicine Department, Benha University, Benha 13115, Egypt. ${ }^{2}$ Biochemistry, Faculty of Medicine, Benha University, Benha 13115, Egypt. ${ }^{3}$ Rheumatology, Benha Teaching Hospital, Benha 13115, Egypt 
Received: 14 May 2020 Accepted: 18 June 2020

Published online: 02 November 2020

\section{References}

1. Smith ML, Long DS, Damiano ER, Ley K (2003) Near-wall micro-PIV reveals a hydrodynamically relevant endothelial surface layer in venules in vivo. Biophys J 85:637-645

2. Béchard D, Gentina T, Delehedde M, Scherpereel A, Lyon M, Aumercier M et al (2001) Endocan is a novel chondroitin sulfate/dermatan sulfate proteoglycan that promotes hepatocyte growth factor/scatter factor mitogenic activity. J Biol Chem 276:48341-48349

3. Pattanaik D, Brown M, Postlethwaite BC, Postlethwaite AE (2015) Pathogenesis of systemic sclerosis. Front Immunol 6:272

4. Castro SV, Jimenez SA (2010) Biomarkers in systemic sclerosis. Biomark Med 4:133-147

5. Jackobsson L, ClaessonWelsh L (2008) Vascular basement membrane components in angiogenesis - an act of balance. Sci World J 8:1246-1249

6. van den Hoogen F, Khanna D, Fransen J, Johnson SR, Baron M, Tyndall A et al (2013) 2013 classification criteria for systemic sclerosis: an American College of Rheumatology/European League against Rheumatism collaborative initiative. Arthritis Rheum 65:2737-2747

7. Davatchi F, Chams-Davatchi C, Ghodsi Z, Shahram F, Nadji A, Shams H et al (2011) Diagnostic value of pathergy test in Behcet's disease according to the change of incidence over the time. Clin Rheumatol 30:1151-1155

8. Aletaha D, Neogi T, Silman AJ, Funovits J, Felson DT, Bingham COIII et al (2010) 2010 rheumatoid arthritis classification criteria: an American College of Rheumatology/European League Against Rheumatism collaborative initiative. Ann Rheum Dis 69:1580-1588

9. Valentini G, Della Rossa A, Bombardieri S, Bencivelli W, Silman AJ, D’Angelo S et al (2001) European multicentre study to define disease activity criteria for systemic sclerosis. II. Identification of disease activity variables and development of a preliminary activity index. Ann Rheum Dis 60:592-598

10. Lawton G, Bhakta BB, Chamberlain MA, Tennant A (2004) The Behçet's disease activity index. Rheumatology (Oxford) 43:73-78

11. Prevoo ML, van't Hof $M A$, Kuper $H H$, van Leeuwen $M A$, van de Putte $L B$, van Riel PL (1995) Modified disease activity scores that include twenty-eight joints counts. Development and validation in a prospective longitudinal study of patients with rheumatoid arthritis. Arthritis Rheum 38:44-48

12. Hassan WA, Hamaad GA, Sayed EA, El Behisy MM, Gomaa MK (2019) Clinical significance of interleukin 27 serum concentration in patients with systemic sclerosis: relation to clinical, laboratory and radiological parameters. Egypt Rheumatol Rehabil 46:101-107

13. Mosevoll KA, Linda SR, Wendelbo O, Bruserud O, Reikvam H (2014) Systemic levels of the endothelium-derived soluble adhesion molecules endocan and Eselectin in patients with suspected deep vein thrombosis. Springerplus 3:571

14. Adekola H, Romero R, Chaemsaithong P, Korzeniewski S, Dong Z, Yeo L, Hassan SS, Chaiworapongsa T (2014) Endocan, a putative endothelial cell marker, is elevated in preeclamp- sia, decreased in acute pyelonephritis, and unchanged in other obstetrical syndromes. J Matern Fetal Neonatal Med 28: $1-12$

15. Sarrazin S, Adam E, Lyon M, Depontieu F, Motte V, Landolfi C et al (2006) Endocan or endothelial cell specific molecule-1 (ESM-1): a potential novel endothelial cell marker and a new target for cancer therapy. Biochim Biophys Acta 1765:25-37

16. Ciftci GG, Ciftci IH, Karakece E, Harman H, Kamanlı A, Tekeoglu I (2016) Identification of ESM-1 as a new endothelial biomarker in the pathogenesis of rheumatoid arthritis. Biotechnol Biotechnol Equip 30:548-552

17. Kul A, Ateş O, Alkan Melikoğlu M, Uğur M, Öztürk N, Erkayhan G, Koçer I (2017) Endocan measurement for active Behçet disease. Arch Rheumatol 32: 197-202

18. Bălănescu P, Lădaru A, Bălănescu E, Voiosu T, Băicuş C, Dan GA (2016) Endocan, novel potential biomarker for systemic sclerosis: Results of a pilot study. J Clin Lab Anal 30:368-373

19. Celık T, Balta S, Karaman M, Ahmet Ay S, Demırkol S, Ozturk C et al (2015) Endocan, a novel marker of endothelial dysfunction in patients with essential hypertension: comparative effects of amlodipine and valsartan. Blood Press 24:55-60

20. Tok D, Ekiz F, Basar O, Coban S, Ozturk G (2014) Serum endocan levels in patients with chronic liver disease. Int J Clin Exp Med 7:1802-1807

21. Cox LA, van Eijk LT, Ramakers BP, Dorresteijn MJ, Gerretsen J, Kox M Pickkers $P$ (2015) Inflammation-induced increases in plasma endocan levels are associated with endothelial dysfunction in humans in vivo. Shock. 43(4): 322-326

22. Aciksari G, Kavas M, Atici A et al (2018) Endocan levels and endothelial dysfunction in patients with sarcoidosis. Angiology 69:878-883

\section{Publisher's Note}

Springer Nature remains neutral with regard to jurisdictional claims in published maps and institutional affiliations.

\section{Submit your manuscript to a SpringerOpen ${ }^{\circ}$ journal and benefit from:}

- Convenient online submission

- Rigorous peer review

- Open access: articles freely available online

High visibility within the field

- Retaining the copyright to your article

Submit your next manuscript at $\boldsymbol{\nabla}$ springeropen.com 